\title{
Zur Prototropie des Fuchsins
}

\section{Gleichgewichte und Reaktionsgeschwindigkeiten in sauren Lösungen}

\author{
Von Waldemar Broser und Robert Havemann \\ Aus dem Kaiser-Wilhelm-Institut für physikalische Chemie und Elektrochemie, Berlin-Dahlem \\ (Z. Naturforschg. 5 a, 385-392 [1950]; eingegangen am 6. Juni 1950)
}

\begin{abstract}
Die Absorptionsspektren wäßriger Fuchsinlösungen zwischen 220 und $280 \mathrm{~m} \mu$ werden bei den $p_{\mathrm{H}}$-Werten 4,$5 ; 1,3$ und 0 aufgenommen. Weiter werden die Gleichgewichte und Reaktionsgeschwindigkeiten der höher geladenen Fuchsin-Kationen bei $20^{\circ} \mathrm{C}$ optisch gemessen. Es ergibt sich, daß sich das Gleichgewicht zwischen dem zweifach geladenen Kation $\left(\mathrm{FH}^{+}+\right)$und dem einfach geladenen $\left(\mathrm{F}^{+}\right)$unmeßbar schnell einstellt. Die Säuredissoziationskonstante beträgt $3,3 \cdot 10^{-\mathbf{2}} \mathrm{mol} / l$. Die Bildungsgeschwindigkeit des dreifach geladenen Kations $\left(\mathrm{FH}_{2}{ }^{3+}\right)$ aus $\mathrm{FH}++$ ist der Messung zugänglich. Die Messung ergibt, daß das höher geladene Kation nicht durch Aufnahme eines Protons, sondern durch Hydroxylionenabspaltung eines durch Protonbrücken verknüpften Hydrates $\mathrm{FH} \cdot \mathrm{H}_{2} \mathrm{O}++$ gebildet wird. Die Säuredissoziationskonstante dieser Reaktion ergibt sich zu 2,09·10-4 $\mathrm{mol} / l$. Die Geschwindigkeitskonstanten betragen $0,63 \min ^{-1}$ für die Hin- und $1,35 \cdot 10^{10} l \cdot \mathrm{mol}^{-1} \mathrm{~min}^{-1}$ für die Rückreaktion. Aus den Zahlenwerten der Dissoziationskonstanten folgt, daß $\mathrm{F}+$ gegenüber $\mathrm{FH}++$ eine weitaus schwächere Base ist als $\mathrm{FH}++$ gegenüber $\mathrm{FH}_{2}{ }^{3+}$ und daß $\mathrm{FH}++$ im totalen Gleichgewicht bei jedem $p_{\mathrm{H}}$-Wert nur in sehr geringem Ausmaße (max. etwa 5\%) vorhanden ist. Diese Befunde stellen weitere experimentelle Beweise für die Mesomerie der Fuchsin-Kationen dar.
\end{abstract}

\section{Theoretische Grundlagen}

1. Die chemische Konstitution der Triphenylmethanfarbstoffe ist lange Zeit hindurch umstritten gewesen, nicht zuletzt deshalb, weil die älteren chemischen Vorstellungen es nicht erlaubten, die Farbänderung zu erklären, die bei ihren Säure-BaseReaktionen oder - wie man neuerdings auch sagt Prototropien ${ }^{1}$ auftreten. Erst in neuerer Zeit konnte eine befriedigende Lösung gefunden werden ${ }^{2}$. Danach zeigt z. B. das Parafuchsin-Kation Mesomerie, d. h. seine Konstitution läßt sich nicht durch eine Formel wiedergeben, sondern nur durch die Grenzformeln

$$
\text { I } \longleftrightarrow \text { II } \longleftrightarrow \text { III } \longleftrightarrow \text { IV }
$$

eingrenzend beschreiben. Hierin sind
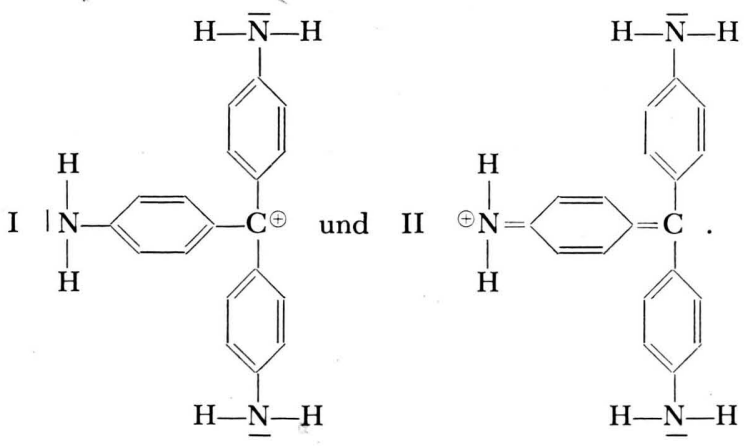

III und IV unterscheiden sich von II dadurch, daß jeweils ein anderer Anilinrest chinoid formuliert wird.
Die Grenzformeln II, III und IV sind zu gleichen Teilen, die Carbenium-Grenzformel I aus energetischen Gründen ${ }^{3}$ dagegen nur in untergeordnetem $\mathrm{Maße}$ an der Mesomerie beteiligt. Wie quantenmechanische Überlegungen ergeben, besitzt das mesomere Kation eine geringere innere Energie als sie jeder der Grenzformeln zukäme, und zwar wird unter sonst gleichen Bedingungen der Energiegewinn um so größer, je mehr Grenzformeln an der Mesomerie beteiligt sind ${ }^{4}$.

Das Methankohlenstoffatom und die 1.4-Achsen der Anilinreste liegen in einer Ebene, letztere schließen gegeneinander einen Winkel von $120^{\circ}$ ein. Die Benzolringe selbst sind aus sterischen Gründen etwas aus dieser Ebene herausgedreht, so daß das Kation etwa die Gestalt eines dreiflügeligen Propellers besitzt 5 .

Das obige Formelschema gibt die chemische Konstitution im Grundzustand wieder. Durch Energieaufnahme können daraus angeregte Zustände hervorgehen, bei denen die "Gewichte“ der einzelnen Grenzformeln anders verteilt sind als im Grundzustand, in dem sie etwa Mesomerien entsprechen, an denen nur ein oder zwei Anilinreste beteiligt sind oder die allein durch die Grenzformeln II oder I beschrieben werden können. Die Anregung kann u. a. durch Licht erfolgen. Die Absorptionsspektren geben also Aufschlüsse über die einzelnen Zustände.

1 Vgl. B. Eistert, Chemismus und Konstitution, Verlag Ferd. Enke, Stuttgart 1948, I. Bd., S. 186.

2 Vgl. W.H ü ckel, Theoret. Grundlagen organ. Chem., 5. Aufl., Akad. Verlagsges. Leipzig 1948, II. Bd., S. 41 ff.

3 Vgl. G. W. Whel and, Theory of Resonance, 4. Aufl., Verlag John Wiley, New York 1947, S. 16.

4 Vgl. T. Förster, Z. Elektrochem. angew. physik. Chem. 45, 548 [1939].

5 Vgl. B. E is t e r t ${ }^{1}$, S. 125 
Wie jede chemische Reaktion verläuft auch die Prototropie über angeregte Zustände. So dürfte die Bildung des zweifach geladenen Kations durch Anlagerung eines Protons an ein N-Atom über einen angeregten Zustand verlaufen, an dessen Mesomerie nur zwei Anilinreste beteiligt sind. Entsprechendes gilt für die Bildung des dreifach geladenen Kations. Die Bildung des vierfach geladenen Kations erfolgt dagegen über die Carbenium-Grenzformel des dreifach geladenen Kations, die also als Reaktionsformel angesehen werden kann. Bei der Bildung von Pararosanilin fungiert hingegen I als Reaktionsformel, in dem sich Hydroxylionen in die Oktettlücke am Methankohlenstoff einlagern.

Die soeben erwähnten Umwandlungen äußern sich auch im Spektrum, da jeder der Verbindungen individuelle Grundzustände und angeregte Zustände zukommen, d. h., das Absorptionsspektrum ist - wie seit langem bekannt vom $p_{\mathrm{H}}$-Wert abhängig.

2. In der vorliegenden Arbeit und den folgenden Veröffentlichungen soll über Untersuchungen berichtet werden, die sich mit den Reaktionen befassen, die das als typischer Vertreter der einfachen basischen Triphenylmethanfarbstoffe gewählte Fuchsin ${ }^{6}$ bei $p_{\mathrm{H}^{-}}$ Änderungen erleidet. Dabei soll neben der Aufnahme der Absorptions s pektren das größte Gewicht gelegt werden auf die Ermittlung der Existenzbereiche der einzelnen Verbindungen, d.h. auf die Bestimmung der einzelnen Gleichgewichts konstanten und auf die Untersuchung der bei den Umwandlungen auftretenden Reaktionsges chwindigkeiten.

Letztere gestatten, Aussagen über den Chemismus der Reaktion zu machen. Die Temperaturabhängigkeit der Gleichgewichts- und Geschwindigkeitskonstanten erlaubt, die Reaktions- und Aktivierungsenergien und -entropien zu berechnen, die gewisse Aufschlüsse über die auf das Vorliegen der Mesomerie zurückzuführenden energetischen und sterischen Effekte geben.

3. Den Untersuchungen werden kurz die wichtigsten the or e tis chen Grundlagen vorangestellt, die Einfluß auf deren Durchführung haben.

a) Für monochromatisches Licht und hinreichend verdünnte Lösungen gilt das $\mathrm{L}$ a m b e r t - B e e r sche Gesetz

$$
\log \frac{J_{0}}{J} \equiv E=\varepsilon c d
$$

$\left(\boldsymbol{J}_{0}=\right.$ Intensität des in die Lösung eintretenden Lichtes; $\boldsymbol{J}=$ Intensität des aus der Lösung austretenden Lichtes; $\boldsymbol{E}=$ Extinktion; $\varepsilon=$ Extinktionskoeffizient $; c=$ Konzentration der absorbierenden Substanz in $\mathrm{mol} / \mathrm{l} ; d=$ Schichtdicke in $\mathrm{cm}$.)

Bei Kenntnis von $\varepsilon$ und $d$ läßt sich aus $E$ also $c$ ermitteln.

6 Das Fuchsin-Kation unterscheidet sich von dem des oben angeführten Parafuchsins durch die Anwesenheit einer Methylgruppe an einem Benzolkern in 3-Stellung. b) Die Prototropie wird definitionsgemäß als eine Umsetzung mit Protonen aufgefaßt. Für die Prototropien z. B. des einfach geladenen mesomeren Fuchsin-Kations, das im folgenden mit $\mathrm{F}^{+}$abgekürzt wird, ergeben sich folgende Schemata

$$
\begin{aligned}
& \mathrm{F}^{+}+\mathrm{H}^{+} \rightleftarrows \mathrm{FH}^{++} \\
& \mathrm{F}^{+}+\mathrm{H}_{2} \mathrm{O} \rightleftarrows \mathrm{FOH}+\mathrm{H}^{+} .
\end{aligned}
$$

$\mathrm{FH}++$ stellt dabei das zweifach geladene Kation, FOH das Rosanilin dar.

Wird während des Ablaufs jedes Versuches die $\mathrm{H}^{+}$Konzentration konstant gehalten, so folgt aus dem MWG 7:

$$
\begin{gathered}
\frac{[F]}{[F H]}=\frac{K_{\mathrm{s}}}{h} \equiv \Re_{\mathrm{s}}, \\
\frac{[F]}{[F O H]}=K_{\mathrm{b}} h \equiv \Re_{\mathrm{b}} .
\end{gathered}
$$

Für die $p_{\mathrm{H}}$-Abhängigkeit der $\Omega$-Werte gilt daher

$$
\begin{aligned}
& \log \Re_{\mathrm{s}}=p_{\mathrm{H}}+\log K_{\mathrm{s}}=p_{\mathrm{H}}-p_{K_{\mathrm{s}}}, \\
& \log \Re_{\mathrm{b}}=-p_{\mathrm{H}}+\log K_{\mathrm{b}}=-p_{\mathrm{H}}-p_{K_{\mathrm{b}}} .
\end{aligned}
$$

Eine Auftragung der gemessenen $\Re$-Werte im logarithmischen Maßstab gegen $p_{\mathrm{H}}$ ergibt daher Geraden der Steigung 1 bzw. -1 . Für $\log \Omega=0$ ergibt sich

$$
\begin{aligned}
& p_{K_{\mathrm{s}}}=p_{\mathrm{H}}, \\
& p_{K_{\mathrm{b}}}=-p_{\mathrm{H}} .
\end{aligned}
$$

Zur Kenntnis der $\mathfrak{R}$-Werte gelangt man auf optischem Wege. Da $E$ eine additive Größe ist, folgt unter Beachtung der Stöchiometrie, wenn alle Versuche bei einer beliebigen, aber stets gleichen Fuchsin-Bruttokonzentration durchgeführt werden,

$$
\begin{aligned}
& \Re_{\mathrm{s}}=\frac{[E]-E_{\mathrm{FH}}}{E_{\mathrm{F}}-[E]}, \\
& \Re_{\mathrm{b}}=\frac{[E]-E_{\mathrm{FOH}}}{E_{\mathrm{F}}-[E]}
\end{aligned}
$$

$\left(E_{\mathrm{FH}}, E_{\mathrm{F}}, E_{\mathrm{FOH}}=\right.$ Extinktion der Lösungen, wenn die betr. Substanzen allein in ihnen vorhanden sind. $[E]=\mathbf{E x}-$ tinktion der Lösung beim Gleichgewicht. Schichtdicke und Farbstoffkonzentration sind die gleichen wie oben.)

In gewissen Fällen ist es zweckmäßig, statt $E$ die auf $E_{\mathrm{F}}=1$ bezogenen Größen $\mathbb{E}=E / E_{\mathrm{F}}$ zu verwenden.

Wie man unschwer erkennt, ergibt eine Auftragung von [๕્] gegen $p_{\mathrm{H}}$ in beiden Fällen S-förmige Kurven, deren Wendepunkte sich bei $p_{\mathrm{H}}=p_{K_{\mathrm{s}}}$ bzw. bei $p_{\mathrm{H}}$ $=-p_{K_{\mathrm{b}}}$ befinden und deren Äste sich asymptotisch 1 und $\mathfrak{F}_{\mathrm{FH}}$ bzw. $\mathfrak{\xi}_{\mathrm{FOH}}$ nähern.

7 Die kursiven Symbole ohne Ladungszeichen in [] bedeuten die Gleichgewichtskonzentrationen der betreffenden Substanzen, $h$ die während jedes Einzelversuchs konstant gehaltene Wasserstoffionenkonzentration. $K_{\mathrm{s}}$ bzw. $1 / K_{\mathrm{h}}$ sind die Säuredissoziationskonstanten. 
Während die Schemata (I) und (II) die Gleichgewichte völlig eindeutig beschreiben, sagen sie dagegen nichts über den Reaktionsweg aus. Eine Entscheidung, ob die Umsetzungen gemäß (I) und (II) oder nach

$$
\begin{aligned}
& \mathrm{F}^{+}+\mathrm{H}_{2} \mathrm{O} \rightleftarrows \mathrm{FH}^{++}+\mathrm{OH}^{-}, \\
& \mathrm{F}^{+}+\mathrm{OH}^{-} \rightleftarrows \mathrm{FOH}
\end{aligned}
$$

verlaufen, ist nicht möglich, da das MWG unter Berücksichtigung des Dissoziationsgleichgewichtes des Wassers ebenfalls zu Gl. (2) führt. Hingegen gestattet die Untersuchung der Reaktionsgeschwindigkeiten eine solche Unterscheidung.

c) Als Z eitge setze erhält man für einen Ablauf nach (I) bzw. (II) 8

$$
\begin{aligned}
& -\frac{d F}{d t}=k_{\mathrm{s} 1} H \cdot F-k_{\mathrm{s} 2} F H, \\
& -\frac{d F}{d t}=k_{\mathrm{b} 1} F \quad-k_{\mathrm{b} 2} H \cdot F O H
\end{aligned}
$$

oder für die Reaktion nach (III) bzw. (IV)

$$
\begin{aligned}
& -\frac{d F}{d t}=k_{\mathrm{s} 1}^{\prime} F \quad-k_{\mathrm{s} 2}^{\prime} \frac{K_{\mathrm{w}}}{H} F H, \\
& -\frac{d F}{d t}=k_{\mathrm{b} 1}^{\prime} \frac{K_{\mathrm{w}}}{H} F-k_{\mathrm{b} 2}^{\prime} F O H .
\end{aligned}
$$

Verlaufen die einzelnen Reaktionen bei konstantem $p_{\mathrm{H}^{-}}$ Wert, so kann $h$ in die Geschwindigkeitskonstanten einbezogen werden, was durch Verwendung von Frakturbuchstaben angedeutet wird. Dadurch erhält man ein Zeitgesetz erster Ordnung. Die Integration der obigen Differentialgleichungen führt dann zu

$$
\mathfrak{f}_{1}+\mathfrak{f}_{2} \equiv \mathfrak{t}=\frac{2,303}{t} \log \frac{F_{0}-[F]}{F-[F]},
$$

oder, wenn die Konzentrationen auf optischem Wege ermittelt werden, zu

$$
\log (E-[E])=-\frac{\mathfrak{t}}{2,303} t+\log \left(E_{\mathrm{F}}-[E]\right)
$$

Geht man also von Lösungen aus, deren $p_{\mathrm{H}}$-Wert derart gewählt ist, daß das Gleichgewicht ganz auf der Seite des $\mathrm{F}+$ liegt, wo $F_{0}$ also gleich der Bruttokonzentration des Fuchsins ist, und bringt diese dann auf einen $p_{\mathrm{H}}$-Wert, dem die Gleichgewichtskonzentration $[F]$ bzw. die Gleichgewichtsextinktion [E] entspricht, so ergibt eine Auftragung von $\log (E-[E])$ gegen $t$ eine Gerade, aus deren Steigung unschwer $\mathfrak{z u}$ berechnen ist ${ }^{9}$. Da ferner $\mathfrak{f}_{2} / \mathfrak{f}_{1}=\Re$, lassen sich $\mathfrak{f}_{2}$ und $\mathfrak{f}_{1}$ getrennt berechnen.

4. Die vorliegende Arbeit befaßt sich mit den mehrfach geladenen Fuchsin-Kationen.

8 Die kursiven Symbole ohne Ladungszeichen bedeuten die laufenden Konzentrationen der betreffenden Substanzen.

9 Vgl. A. S k r a b a l, Homogenkinetik, Verlag Th. Steinkopff, Dresden u. Leipzig 1941, S. 99.

10 J. S chmidlin, C. R. hébd. Séances Acad. Sci. 138, 1508 [1904].
Von den mehrsäurigen Salzen des Fuchsins konnten bisher nur dreisäurige und viersäurige Salze isoliert werden. So erhielten J. S c h midlin 10 und vor ihm A. W. $\mathrm{H}$ of $\mathrm{m}$ a n $\mathrm{n}^{11}$ das Fuchsintrichlorid als schwarzes, grünstichiges Pulver, und K. A. H of m a n n 12 das Fuchsintetraperchlorat als dunkeloranges bis kupferfarbenes Kristallpulver. Die wäßrigen Lösungen beider Substanzen zeigten infolge Hydrolyse die gleiche Färbung wie die des einsäurigen Salzes.

Über Farbänderungen, die beim Versetzen der roten Parafuchsin- bzw. Fuchsinlösungen mit Säuren auftreten, haben F. Kehrmann und M. Sandoz sowie P. Rumpf $\mathbf{1 4}$ berichtet. Danach entstehen je nach der Stärke der verwendeten Säure rotviolette oder gelbe Lösungen, die sich nach einiger Zeit entfärben. Bei Anwendung sehr starker Säuren ist jedoch die gelbe Farbe beständig. Kehrmann und Sandoz schreiben die rotviolette Färbung dem zweisäurigen, die unbeständige gelbe dem dreisäurigen, die beständige gelbe Färbung dem viersäurigen Salz und die farblose Lösung dem „Carbinol-Salz“ zu.

Unbeschadet dieser sicher nicht ganz richtigen Interpretierung lassen die mitgeteilten Ergebnisse das Vorliegen verschiedener Gleichgewichte erwarten, deren zeitliche Einstellung meßbar ist.

Im folgenden wird über die Messung der Fuchsinspektren bei den $p_{\mathrm{H}}$-Werten 4,$5 ; 1,3$ und 0 sowie über quantitative Untersuchungen der Gleichgewichte und der Reaktionsgeschwindigkeiten der höhergeladenen Fuchsin-Kationen berichtet. Zur Erzielung einfacher Verhältnisse werden die Wasserstoffionenkonzentrationen während jedes Einzelversuches konstant gehalten und alle Versuche bei der Ionenstärke 0,1 durchgeführt. Vorversuche ergaben, daß bei $p_{\mathrm{H}} 5$ die Gleichgewichte praktisch völlig auf der Seite des $\mathrm{F}^{+}$liegen. Somit ergibt sich, daß sich die Untersuchungen auf den $p_{\mathrm{H}^{-}}$ Bereich zwischen 1 und 5 erstrecken.

\section{Beschreibung der Versuche}

\section{Versuchs anordnung}

a) Zur Aufnahme der Absorptionsspektren und zur optischen Konzentrationsbestimmung diente die von R. H a v e m a n n u. P. Wo lf ${ }^{15}$ beschriebene Apparatur.

Monochromatisches Licht, das durch Zerlegung des Lichtes einer geeigneten Strahlungsquelle in einem Quarzdoppelmonochromator hergestellt wird, kann wahl-

11 A. W. Hofmann, Proc. Roy. Soc. [London], Ser. A 12, 7 [1862]

12 K. A. Hofma n n, R. R oth, K. Höbold u.

A. Metzl e r, Ber. dtsch. chem. Ges. 43, 2624 [1910].

13 F. K e h rm a n n u. M. S a n d o z, Ber. dtsch. chem.

Ges. 51, 915 [1918].

14 P. Rum pf, Ann. Chimie [11] 3, 327 [1935].

15 R. $\mathrm{H}$ avem a n $\mathrm{n}$. P. Wolf, im Erscheinen. 
weise durch Küvetten, die die Meßlösung oder die NullLösung enthalten, geschickt werden. Die Küvetten befinden sich in einem temperierten Kasten. Die Intensität der aus den Küvetten austretenden Strahlen wird mit einer Sekundärelektronenvervielfacher-Photozelle gemessen. Die vervielfachten Photoströme sind der Lichtintensität proportional. Die Null-Lösung hat bis auf den Farbstoff die gleiche Zusammensetzung wie die Meßlösung, so daß die Absorption des Lösungsmittels und der Lösungsgenossen eliminiert, also die Extinktionen lediglich des Farbstoffes erhalten werden, wenn für $J_{0}$ die der Null-Lösung, für $\boldsymbol{J}$ die der Meßlösung entsprechenden Galvanometerausschläge eingesetzt werden. Die große Empfindlichkeit der Sekundärelektronenvervielfacher-Photozelle bringt es mit sich, daß die Messungen bei außerordentlich kleinen Lichtintensitäten durchgeführt werden, Störungen durch photochemische Prozesse also nicht $\mathrm{zu}$ erwarten sind.

b) Die bei den Messungen verwendete Fuchsinlösung wurde aus besonders gereinigtem Rosanilin hergestellt.

Käufliches Diamantfuchsin wird mit 1-n. Natronlauge etwa 1 Stde. rückgekocht, die hellfarbige Lösung noch heiß durch ein Filter gegeben. Die nach dem Erkalten ausgeschiedenen farblosen Rosanilin-Kristalle werden rasch abgenutscht, mit verd. Natronlauge gewaschen und dann in wenig Wasser, dem etwas Salzsäure zugesetzt ist, aufgenommen. Aus dieser Lösung wird mit Natronlauge, wie oben, erneut Rosanilin gefällt. Die nach zweimaliger Wiederholung des Verfahrens erhaltenen Rosanilin-Kristalle werden im Vakuum über Natriumhydroxyd bei etwa $50^{\circ} \mathrm{C}$ scharf getrocknet. 0,319 $\mathrm{g} \triangleq 0,001 \mathrm{~mol}$ dieses Rosanilins werden abgewogen und zusammen mit $10 \mathrm{~cm}^{3} \quad 0,1-n$. Salzsäure mit Wasser bei $20^{\circ} \mathrm{C} \mathrm{zu}$ einem Liter aufgefüllt. Der $p_{\mathrm{H}^{-}}$Wert dieser Lösung wurde zu ungefähr 3,8 bestimmt.

Durch Verdünnen dieser Lösung unter Hinzufügen einer kleinen Menge Acetatpuffer $p_{\mathrm{H}}$ 4,5 wurde eine schwachgepufferte Farblösung (im folgenden mit D III p bezeichnet) des $p_{\mathrm{H}} 4,5$ und der Fuchsin-Konzentration $2,5 \cdot 10^{-4} \mathrm{~mol} / l$ erhalten. Von dieser Lösung, in der, wie die Versuche ergaben, die Gleichgewichte praktisch völlig auf seiten von $\mathrm{F}^{+}$liegen, wurde im folgenden stets ausgegangen.

c) Als Puffer fanden Glykokoll-, Phosphorsäureund Acetatpuffer Verwendung. Es wurde darauf geachtet, daß die Ionenstärke aller Puffer 0,2 betrug. Die Meßlösungen, die daraus durch Verdünnen 1:1 mit Farblösung-Wassermischungen hergestellt wurden, hatten also die Ionenstärke 0,1 .

Die Glykokollpuffer wurden nach den im K üs te r Thi e $\mathbf{1 6}$ angegebenen Vorschriften hergestellt, die Konzentrationen der Ausgangslösungen wurden jedoch alle verdoppelt.

16 F. W. K ü s te r, Logarithm. Rechentafeln, 56. bis 60. Aufl., W. de Gruyter, Berlin 1947, S. 157.
Die Phosphorsäure- und Acetatpuffer wurden durch Vermischen von je $10-60 \mathrm{~cm}^{3}$ (entsprechend dem gewünschten $p_{\mathrm{H}}$-Wert) 2-m. Phosphor- bzw. Essigsäure mit je $20 \mathrm{~cm}^{3} 1-n$. Natronlauge und anschließendem Auffüllen auf $100 \mathrm{~cm}^{3}$ hergestellt.

Die genauen $p_{\mathrm{H}}$-Werte der Puffer wurden nach Verdünnen 1:1 mit Wasser potentiometrisch unter Verwendung der Platin-Wasserstoff-Elektrode gemessen. Analoge Mischungen aus Puffer und Farblösung zeigten - wie Vergleichsmessungen mit der Glaselektrode ergaben — dieselben $p_{\mathrm{H}}$-Werte.

\section{Versuchsdurchführung}

a) Die Aufnahme der Abs or ptions spektren erfolgte in der Weise, daß Null-Lösung und Meßlösung bis auf den Farbstoff identische Zusammensetzung hatten. Die Meßlösung wurde an dem der Messung vorangehenden Tage durch Zusammengeben der Fuchsin-Stammlösung mit dem gewünschten Puffer hergestellt und bei $20,0^{\circ} \mathrm{C}$ aufbewahrt; es hatte sich also bestimmt der dem jeweiligen $p_{\mathrm{H}}$ entsprechende Gleichgewichtszustand eingespielt. Die Konzentration der Meßlösung wurde dem jeweiligen $p_{\mathrm{H}}$-Wert und dem Spektralbereich derart angepaßt, daß die gemessenen Extinktionswerte im allgemeinen zwischen 0,1 und 1,0 lagen. Die Gültigkeit des Lambert-Beerschen Gesetzes war bei diesen Konzentrationen hinreichend genau erfüllt. Als Lichtquelle diente im Sichtbaren eine Glühlampe, im UV eine Hg-Spektrallampe. Zur Messung wurden im UV möglichst die Hg-Linien benutzt; wo diese nicht dicht genug lagen, wurde auch im Hg-Kontinuum gearbeitet. In diesem Fall wurde zur Vermeidung von Fremdlicht darauf geachtet, daß die Messungen nicht in der Nähe der Linien durchgeführt wurden.

Abb. 1 zeigt drei charakteristische Spektren. Kurve I gibt das Spektrum bei $p_{\mathrm{H}} 4,5$ (Acetatpuffer), Kurve II bei $p_{\mathrm{H}} 1,3$ (Glykokollpuffer ${ }^{17}$ ) wieder. In beiden Fällen beträgt die Ionenstärke 0,1 . Zum Vergleich ist in Kurve III das Spektrum in 2- $n$. Schwefelsäure $\left(p_{\mathrm{H}} 0\right)$ dargestellt.

Wie man aus Abb. 1 erkennt, ist der Extinktionsunterschied zwischen den Kurven I und II bei $v^{*}=1,83 \cdot 10^{4} \mathrm{~cm}^{-1}$ entspr. $\lambda=546 \mathrm{~m} \mu$ am größten. Die folgenden Messungen wurden deshalb alle bei der Hg-Linie $\lambda=546 \mathrm{~m} \mu$ ausgeführt.

b) Zur Ermittlung der Re a ktions ge s chwindigkeiten und der Gleichgewichte wurde wie folgt verfahren.

17 Wird statt des Glykokollpuffers ein Phosphorsäurepuffer des gleichen $p_{\mathrm{H}}$-Wertes benutzt, so erhält man dasselbe Spektrum; es ist also nicht pufferspezifisch. 
$\mathrm{Zu}$ den in der Meßküvette befindlichen $20 \mathrm{~cm}^{3}$ der gewünschten Pufferlösung wurde zur Zeit 0 rasch die Fuchsinlösung ( $1 \mathrm{~cm}^{3}$ D III p $\left.+19 \mathrm{~cm}^{3} \mathrm{H}_{2} \mathrm{O}\right)$ hinzugegeben. Die Fuchsin-Bruttokonzentration betrug also 6,25 $\cdot 10^{-6} \mathrm{~mol} / l$. Nach $15 \mathrm{sec}$ konnte bereits die erste Absorptionsmessung durchgeführt werden, der sich die

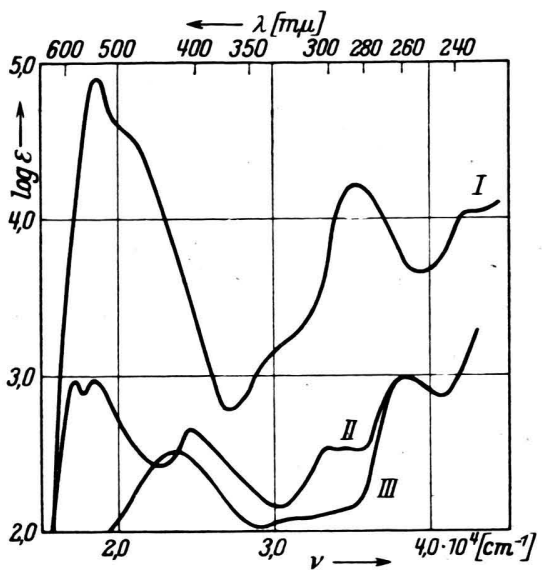

Abb. 1. Fuchsinspektren bei verschiedenen $p_{\mathrm{H}^{-}}$Werten. I: $p_{\mathrm{H}} 4,5$, II: $p_{\mathrm{H}} 1,3$, III: $p_{\mathrm{H}} 0$.

1

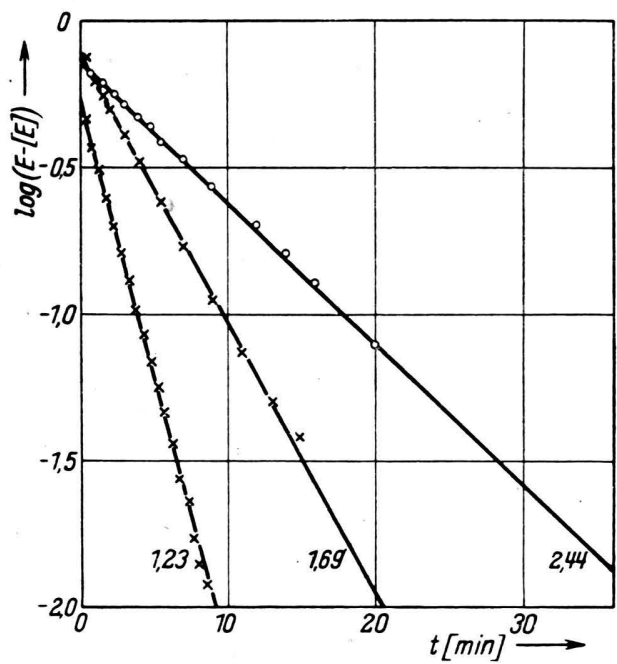

Abb. 2. Darstellung des Reaktionsverlaufs nach Gl. (8).

weiteren Messungen in geeigneten Zeitabständen anschlossen, bis sich konstante Extinktionen eingestellt hatten. Die Temperatur der Lösungen betrug vor dem Zusammengeben $20,0^{\circ} \mathrm{C}$. Diese Temperatur wurde während der ganzen Messung innegehalten. Analog wurde $E_{\mathrm{F}}$ ermittelt. In diesem Fall wurde der Puffer $p_{\mathrm{H}} 4,5$ verwendet; die dabei gemessenen Extinktionen ändern sich dabei natürlich nicht mit der Zeit.

In Abb. 2 sind drei Zeitversuche nach der im Abschnitt I3c angegebenen Art aufgetragen. Wie man

\begin{tabular}{|c|c|c|l|}
\hline$p_{\mathbf{H}}$ & {$[ङ]_{\alpha}$} & {$[\leftleftarrows]_{\sigma}$} & $\mathfrak{k}$ \\
\hline 1,16 & 0,348 & - & 0,500 \\
1,23 & 0,386 & - & 0,430 \\
1,33 & 0,432 & - & 0,382 \\
1,54 & 0,544 & - & 0,265 \\
1,69 & 0,596 & 0,015 & 0,219 \\
1,87 & 0,700 & 0,057 & 0,156 \\
2,21 & 0,870 & 0,189 & 0,1065 \\
2,44 & 0,914 & 0,360 & 0,108 \\
2,60 & 0,935 & 0,497 & 0,0974 \\
2,69 & 0,937 & 0,527 & 0,0913 \\
2,95 & 0,960 & 0,766 & 0,101 \\
\hline
\end{tabular}

Tab. 1.

erkennt, liegen die Meßpunkte - von denen der besseren Übersichtlichkeit wegen nur jeder dritte eingezeichnet wurde - sehr genau auf Geraden, die Reaktionen verlaufen also nach der ersten Ordnung. Die hier nicht gebrachten Zeitkurven für die übrigen $p_{\mathrm{H}}$-Werte zeigen dieselbe Geradlinigkeit und dieselbe geringe Streuung der Meßwerte.

Eine genauere Betrachtung der Kurven führt jedoch zu unerwarteten Ergebnissen. Der Schnittpunkt der Geraden mit der $\log (E-[E])$-Achse sollte es nach Gl. (8) gestatten, $E_{\mathrm{F}}$ auszurechnen, wobei die berechneten Werte zahlenmäßig mit dem bei $p_{\mathrm{H}} 4,5$ beobachteten Wert identisch sein muß. Es zeigt sich aber, daß man stets kleinere Werte erhält, und zwar um so kleinere, je kleiner der $p_{\mathrm{H}}$-Wert ist. Diese errechnete Größe sei mit $[E]_{a}$ bezeichnet, der besseren Unterscheidung halber wollen wir die Extinktion im Gleichgewicht, die oben $[E]$ geschrieben wurde, mit $[E]_{\sigma}$ bezeichnen.

Tab. 1 gibt die aus diesen Versuchen ermittelten $[\mathfrak{E}]_{a},[\mathfrak{E}]_{\sigma}$ und $\mathfrak{f}$-Werte.

\section{Ergebnisse}

1. In Abb. 3 sind unten $\left[[\mathfrak{E}]_{\alpha}\right.$ und $[\tilde{\mathscr{E}}]_{\sigma}$ gegen den $p_{\mathrm{H}}$-Wert aufgetragen, darüber die daraus nach Gl. (5) ermittelten $\Re$-Werte. Es ergeben sich beide Male Geraden. Im ersteren Fall besitzt die Gerade auch, wie in Gl. (3) gefordert, die Steigung 1, im zweiten Fall $\sim 2$. Diese Befunde lassen sich zwanglos durch folgendes Schema deuten

$$
\begin{aligned}
& \mathrm{F}^{+}+\mathrm{H}^{+} \rightleftarrows \mathrm{FH}^{++}, \\
& \mathrm{FH}^{++}+\mathrm{H}^{+} \underset{4}{\stackrel{3}{\rightleftarrows}} \mathrm{FH}_{2}{ }^{3+}
\end{aligned}
$$

bzw. durch eine analoge Folge nach (III). Das Gleichgewicht $\alpha$ stellt sich dabei unmeßbar rasch ein, ist also erreicht, bevor noch die Reaktion $\beta$ zum Zuge 
gekommen ist, d. h. ehe sich $\mathrm{FH}_{2}{ }^{3+}$ gebildet hat. Dadurch ist es möglich, die entsprechenden Gleichgewichtsextinktionen $[E]_{\alpha}$ in der oben angegebenen Weise zu ermitteln.

Während der anschließend einsetzenden langsamen Reaktion $\beta$ bleibt $\alpha$ ständig im Gleichgewicht, ist also laufendes Gleichgewicht geworden ${ }^{18}$. Das gesamte System kommt erst im totalen Gleichgewicht zur Ruhe, d.h. wenn auch $\beta$ das Gleichgewicht erreicht hat. Die Steigung 2 der $\log \Omega_{\sigma}-p_{\mathrm{H}}$-Kurve bedeutet, daß im totalen Gleichgewicht $\mathrm{FH}^{++}$nur in vernach-

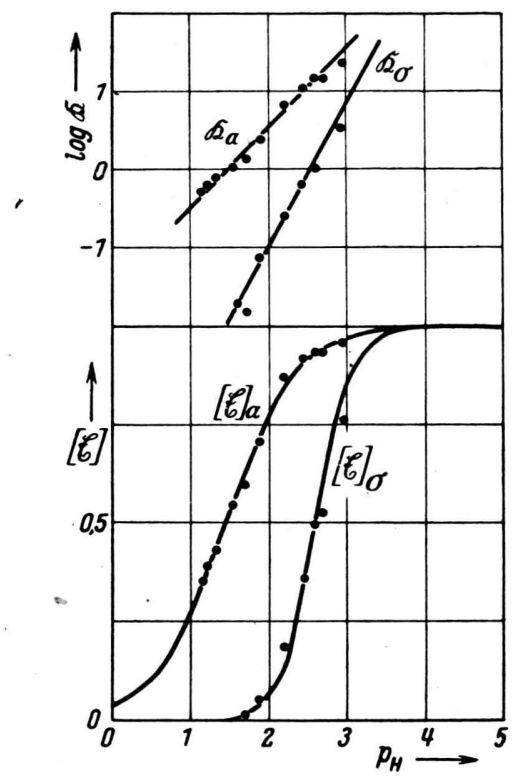

Abb. 3. Die $p_{\mathrm{H}}$-Abhängigkeit der Gleichgewichtsextinktionen und Gleichgewichtskonstanten.

lässigbarem $\mathrm{Maße}$ neben $\mathrm{F}^{+}$und $\mathrm{FH}_{2}{ }^{3+}$ vorkommen kann. Man kommt nämlich zu diesem Faktor, wenn man gemäß der Gesamtreaktion,

$$
\mathrm{F}^{+}+2 \mathrm{H}^{+} \rightleftarrows \mathrm{FH}_{2}{ }^{+},
$$

in der also $\mathrm{FH}^{++}$nicht vorkommt, das MWG ansetzt

$$
\begin{gathered}
\frac{[F]}{\left[F H_{2}\right]}=\frac{K_{\sigma}}{h^{2}} \equiv \mathfrak{\Re}_{\sigma}=\frac{[E]_{\sigma}}{E_{\mathrm{F}}-[E]_{\sigma}}, \\
\log \mathfrak{\Re}_{\sigma}=2 p_{\mathrm{H}}-p_{K_{\sigma}}
\end{gathered}
$$

und für $\log \Re_{\sigma}=0$,

$$
p_{K_{\sigma}}=2 p_{\mathrm{H}} .
$$

Aus $\mathfrak{K}_{\sigma}$ und dem oben bereits erhaltenen

$$
\Re_{\alpha}=\frac{[F]}{[F H]}=\frac{[E]_{\alpha}}{E_{\mathrm{F}}-[E]_{\alpha}},
$$

ergibt sich für

$$
\mathfrak{R}_{\beta}=\frac{[F H]}{\left[F H_{2}\right]}=\frac{\mathfrak{\Re}_{\sigma}}{\mathfrak{\Re}_{\alpha}} .
$$

Aus Abb. 3 erhält man gemäß Gl. (4) die Zahlenwerte

$$
\begin{aligned}
& p_{K_{\alpha}}=1,48 \text { entspr. } K_{\alpha}=3,30 \cdot 10^{-2} \mathrm{~mol} / l, \\
& p_{K_{\sigma}}=5,16 \text { entspr. } K_{\sigma}=6,90 \cdot 10^{-6}(\mathrm{~mol} / l)^{2}
\end{aligned}
$$

und weiter nach Gl. $\left(2, \mathrm{I}_{\beta}\right)$

$$
p_{K \beta}=3,68 \text { entspr. } K_{\beta}=2,08 \cdot 10^{-4} \mathrm{~mol} / l .
$$

Während die Steigung der $\log \Re_{\alpha}-p_{\mathrm{H}}$-Kurve genau 1 ist, beträgt der genaue Wert $\operatorname{der} \log \Re_{\sigma}-p_{\mathrm{H}}$-Kurve nicht 2 , sondern nur 1,76. Diese Abweichung dürfte darauf zurückzuführen sein, daß die, wenn auch in sehr geringem Maße vorhandenen $\mathrm{FH}++$ - und $\mathrm{FH}_{3}{ }^{4+}$-Kationen einen Beitrag zur Lichtabsorption liefern, der vom $p_{\mathrm{H}}$-Wert in unbekannter Weise abhängt und der deshalb in der Rechnung nicht berücksichtigt werden konnte. Der hierdurch verursachte Fehler ist aber bei ungefähr hälftiger Umwandlung, also bei $p_{\mathrm{H}} 2,58$ relativ am kleinsten, so daß die oben angwendete Methode zur Ermittlung von $\boldsymbol{K}_{\sigma}$ hinreichend genau ist.

2. Das $\mathrm{Z}$ e it g e s e tz der Reaktion $\alpha$ kann wegen der unmeßbar großen Reaktionsgeschwindigkeit nicht ermittelt werden. Für das Zeitgesetz der langsameren Reaktion $\beta$ gilt, wie festgestellt, Gl. (8) mit der Maßgabe, daß $[E]_{a}$ an Stelle von $E_{\mathrm{F}}$ tritt. Aus den bereits ermittelten Werten von $\mathfrak{f}$ müssen nun unter Berücksichtigung des vorgelagerten Gleichgewichtes $\alpha$ die Geschwindigkeitskonstanten der Reaktion $\beta$ berechnet werden.

Das Zeitgesetz der Reaktion $\beta$ lautet

$$
-\frac{d F H}{d t}=\mathfrak{f}_{3} F H-\mathfrak{f}_{4} F_{2} .
$$

Die der Messung zugängliche Größe $F$ kommt hierin nicht vor, sondern muß erst über das laufende Gleichgewicht $\alpha$ eingeführt werden, was zur Gleichung

$$
-\frac{d E}{d t}=\left(\mathfrak{f}_{4}+\mathfrak{f}_{3} \frac{E_{\mathrm{F}}-[E]_{\alpha}}{E_{\mathrm{F}}}\right)+\mathfrak{k}_{4}[E]_{\alpha}
$$

führt. Bei $d E / d t=0$ herrscht totales Gleichgewicht, d. h. $E=[E]_{\sigma}$, ferner gilt $\mathfrak{f}_{4} / \mathfrak{f}_{3}=\mathfrak{\Re}_{\beta}$. Mit

folgt daher

$$
\mathfrak{k}_{4}+\mathfrak{k}_{3} \frac{E_{\mathrm{F}}-[E]_{\alpha}}{E_{\mathrm{F}}}=\mathfrak{k}
$$

und

$$
\mathfrak{k}_{4}=\mathfrak{f} \frac{[E]_{\sigma}}{[E]_{\alpha}}
$$

$$
\mathfrak{f}_{3}=\mathfrak{f} \frac{\left([E]_{\alpha}-[E]_{\sigma}\right) E_{\mathrm{F}}}{\left(E_{\mathrm{F}}-[E]_{\alpha}\right)[E]_{\alpha}} .
$$

18 Vgl. A. Skrabal ${ }^{9}$, S. 41. 
Durch Integration der Gl. (10) gelangt man zu der oben bereits empirisch gefundenen und benutzten Formel

$$
\log \left(E-[E]_{\sigma}\right)=-\frac{\mathfrak{k}}{2,303} t+\log \left([E]_{\alpha}-[E]_{\sigma}\right) .
$$

In Abb. 4 sind die nach Gl. (13) und (12) berechneten Werte für $\mathfrak{f}_{3}$ und $\mathfrak{f}_{4}$ im logarithmischen Maßstab gegen $p_{\mathrm{H}}$ aufgetragen. Man erkennt, daß $\mathfrak{f}_{3} p_{\mathrm{H}^{-}}$

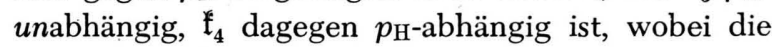
Meßwerte auf einer Geraden der Steigung $\sim 1$ liegen.

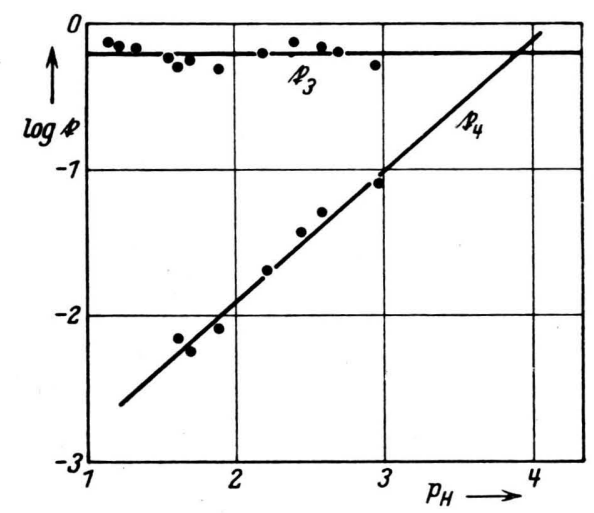

Abb. 4. Die $p_{\mathrm{H}^{-}}$Abhängigkeit der Geschwindigkeitskonstanten.

Es gelten demnach die Beziehungen

$$
\begin{aligned}
& \mathfrak{k}_{3}=k_{3}, \\
& \mathfrak{k}_{4}=k_{4} \frac{K_{\mathrm{w}}}{h} .
\end{aligned}
$$

Die Reaktion $\beta$ ist also gemäß (III)

$$
\mathrm{FH}^{++}+\mathrm{H}_{2} \mathrm{O} \underset{4}{\stackrel{3}{\rightleftarrows}} \mathrm{FH}_{2}{ }^{3+}+\mathrm{OH}^{-}
$$

zu formulieren.

Die Abweichungen bei $\mathfrak{f}_{4}$ von der Steigung 1 gehen auf die Abweichungen bei $\Re_{\sigma}$ zurück, da die diesen entsprechende Größe $[E]_{\sigma}$ in Gl. (12) als Faktor auftritt. Die Meßwerte streuen bei den Geschwindigkeitskonstanten stärker als bei den Gleichgewichtskonstanten, was verständlich ist, da zu ihrer Berechnung drei Meßwerte herangezogen werden mußten.

Aus den Kurven entnimmt man die Zahlenwerte ${ }^{19}$ $p_{\mathrm{k} 3}=\quad 0,2$ entspr. $k_{3}=0,63 \mathrm{~min}^{-1}$, $p_{\mathrm{k} 4}=-10,13$ entspr. $k_{4}=1,35 \cdot 10^{10} l \cdot \mathrm{mol}^{-1} \mathrm{~min}^{-1}$.

19 Als Bezugswert diente analog wie bei $K_{\sigma}$ der $\mathfrak{f}_{4}$-Wert, der für das $p_{\mathrm{H}}$ der hälftigen Umwandlung zu 2,58 interpoliert wurde.
Der sehr große Wert für $k_{4}$ erklärt sich dadurch, daß im Meßbereich die Hydroxylionenkonzentration äußerst gering ist.

3. Die Spektren der Abb. 1 sind nach den obigen Ergebnissen den Kationen $\mathrm{F}^{+}$(Kurve I) und $\mathrm{FH}_{2}{ }^{3+}$ (Kurve II) zuzuordnen.

Während man diese Zuordnung bei Kurve I unbedenklich vornehmen kann, ist bei Kurve II Vorsicht geboten, da die Extinktionen hier relativ gering sind, so daß die Beiträge, die die im Gleichgewicht stets vorhandenen $\mathrm{F}^{+}$ und $\mathrm{FH}^{+}+$liefern, nicht zu vernachlässigen sind. Man erkennt deutlich, daß die bei $\lambda=546 \mathrm{~m} \mu$ liegende Bande des $\mathrm{F}+$ noch beträchtlich ist. Die Kurve III dürfte im wesentlichen auf $\mathrm{FH}_{3}{ }^{4+}$ zurückzuführen sein. Eine Aufnahme des Spektrums von $\mathrm{FH}^{++}$ist nicht möglich, da dieses Kation in keinem $p_{\mathrm{H}}$-Gebiet hinreichend lange beständig ist. Orientierende Versuche durch kinetische Messungen bei verschiedenen Wellenlängen ergaben aber so viel, daß dieser Verbindung eine Bande mit dem Maximum bei $\lambda=580 \mathrm{~m} \mu$ zuzuordnen ist. Dieses Maximum ist auch bei Kurve II deutlich erkennbar.

\section{Folgerungen}

1. Zunächst ist zu erwähnen, daß die Bildung des höhergeladenen Kations nicht durch Aufnahme eines Protons oder, genauer gesagt, durch Reaktion mit einem Hydronium-Kation $\mathrm{H}_{3} \mathrm{O}^{+}$vor sich geht, sondern in einer Dissoziation eines „Hydrates“ besteht. Der Chemismus der Reaktion $\beta$ ist demnach etwa wie folgt aufzulösen:

$$
\begin{aligned}
& \mathrm{FH}^{++} \rightleftarrows\left(\mathrm{FH}^{++}\right)^{*} \\
& \left(\mathrm{FH}^{++}\right)^{*}+\mathrm{H}_{2} \mathrm{O} \rightleftarrows \mathrm{FH} \cdot \mathrm{H}_{2} \mathrm{O}^{++} \\
& \mathrm{FH} \cdot \mathrm{H}_{2} \mathrm{O}^{++} \rightleftarrows \mathrm{FH}_{2}{ }^{3+}+\mathrm{OH}^{-} .
\end{aligned}
$$

Hierin soll $\mathrm{FH}^{++}$den Grundzustand, $(\mathrm{FH}++) *$ einen angeregten Zustand des Kations bedeuten; bei letzterem ist das einsame Elektronenpaar einer der noch freien beiden Aminogruppen nicht an der Mesomerie beteiligt. $\mathrm{Zu}$ , seiner Beschreibung ist also, wie bei dem aus ihm gebildeten $\mathrm{FH}_{2}{ }^{3+}$, die Carbenium- und eine chinoide Grenzformel ausreichend. Das Hydrat $\mathrm{FH} \cdot \mathrm{H}_{2} \mathrm{O}^{++}$entsteht dann unter Ausbildung einer Protonbrücke unter Beteiligung des erwähnten einsamen Elektronenpaares gemäß<smiles>[R]NCO</smiles>

Die Dissoziation dieses Hydrates führt endlich zu dem höher geladenen Kation.

Für einen solchen Verlauf über eine Additionsverbindung spricht nicht nur, daß bei sehr vielen chemischen Reaktionen ähnliche, durch Protonenbrücken verknüpfte „Vorverbindungen“ eine nachweisbar maß- 
gebende Rolle ${ }^{20}$ spielen, sondern auch, daß damit zwanglos zu erklären ist, weshalb die Reaktion nach Schema (III) und nicht nach Schema (I) verläuft.

Bei einem Chemismus nach (I) müßte nämlich die zur Überwindung der elektrostatischen Abstoßungskräfte zwischen den beiden gleichgeladenen Reaktionspartnern $\mathrm{FH}^{+}+$und $\mathrm{H}^{+}$sowie die zur Bildung des angeregten Zustandes $\left(\mathrm{FH}^{++}\right) *$ erforderliche Energie "gleichzeitig“ aufgebracht werden. Bei einem Verlauf nach (III) reagiert das angeregte $\left(\mathrm{FH}^{++}\right)$mit einem der stets in unmittelbarer Nähe vorhandenen ungeladenen Wassermolekülen unter Ausbildung der Protonbrücke, wobei nur ein relativ kleiner Energieberg überwunden werden muß. Dieser so entstandenen, mehr oder minder stabilen Zwischenverbindung kann nun von ihrer Umgebung Energie zugeführt werden, was zur Abdissoziation von Hydroxylionen führt. Der hierbei zu überwindende Energieberg ist ebenfalls kleiner als der bei (I). Der Reaktionsverlauf nach (III) ist also deshalb begünstigt, weil bei ihm infolge der Zwischenverbindung kleinere Aktivierungsenergien auftreten.

2 . Weiter ist beachtenswert, daß Reaktion $\alpha$ in beiden Richtungen viel schneller verläuft als Reaktion $\beta$. Bei der gebotenen Vorsicht hinsichtlich etwaiger Deutungsversuche kann nur so viel gesagt werden, daß die Unterschiede der Reaktionsgeschwindigkeiten letzten Endes durch die Mesomerie bedingt sein werden.

3. Aus den Zahlenwerten für $K_{\alpha}$ und $K_{\beta}$ geht hervor, daß $\mathrm{F}^{+}$gegenüber $\mathrm{FH}^{++}$eine weitaus schwächere Base ist als $\mathrm{FH}^{++}$gegenüber $\mathrm{FH}_{2}{ }^{3+}$. Hierin weicht das Fuchsin von dem Verhalten gewöhnlicher mehrsäuriger Basen ab. Bei diesen ist nämlich hauptsächlich aus elektrostatischen Gründen - B gegenüber $\mathrm{BH}^{+}$eine stärkere $\mathrm{Base}$ als $\mathrm{BH}^{+}$gegenüber $\mathrm{BH}_{2}{ }^{++}$, wobei $\mathrm{B}$ eine mehrsäurige Base, z. B. Benzidin sei. Der Grund für dieses abweichende Verhalten des Fuchsins liegt darin, daß $\mathrm{F}^{+}$, wie eingangs erwähnt, durch die Mesomerie stärker stabilisiert wird als $\mathrm{FH}^{++}$.

${ }^{20}$ Vgl. B. E is te r t ${ }^{1}$, I. Bd., S. 231.
Im einzelnen ergibt sich folgendes:

Zwischen den Gleichgewichtskonstanten und der freien Normalenthalpie $G^{0}$ bestehen die Beziehungen

$$
\begin{aligned}
& R T \ln K_{\alpha}=-\left(G_{\mathrm{F}}^{0}+G_{\mathrm{H}}^{0}-G_{\mathrm{FH}}^{0}\right), \\
& R T \ln K_{\beta}=-\left(G_{\mathrm{FH}}^{0}+G_{\mathrm{H}}^{0}-G_{\mathrm{FH}_{2}}^{0}\right) .
\end{aligned}
$$

Da definitionsgemäß $G_{\mathrm{H}}^{0}=0$, folgt für $20^{\circ} \mathrm{C}$ :

$$
\begin{aligned}
G_{\mathrm{F}}^{0}-G_{\mathrm{FH}}^{0} & =1,34 p_{K_{\alpha}}=1,98 \mathrm{kcal}, \\
G_{\mathrm{FH}}^{0}-G_{\mathrm{FH}_{2}}^{0} & =1,34 p_{K_{\beta}}=4,93 \mathrm{kcal} .
\end{aligned}
$$

Aus dem Vergleich mit den Werten für Benzidin $\left(p_{K \mathbf{1}}\right.$ $\left.=3,65 ; p_{K 2}=4,87\right)$

$$
\begin{aligned}
G^{0}{ }_{\mathrm{B}}-G^{0}{ }_{\mathrm{BH}} & =6,50 \mathrm{kcal}, \\
G^{0}{ }_{\mathrm{B}}-G^{0}{ }_{\mathrm{BH}_{2}} & =4,90 \mathrm{kcal}
\end{aligned}
$$

wird deutlich, daß $G^{0} \mathrm{~F}$ gegenüber dem "Normalfall“ $G^{0}{ }_{\mathrm{B}}$ einen zu kleinen Wert besitzt, was hauptsächlich auf die Erniedrigung der inneren Energie durch die Mesomerie zurückzuführen sein dürfte. Inwieweit noch Entropieeffekte mitspielen, kann erst nach Untersuchung der Temperaturabhängigkeit der Gleichgewichte entschieden werden.

4. Auch der Umstand, daß zweisäurige Salze des Fuchsins nicht isoliert werden konnten, geht letzten Endes auf die Mesomerie zurück, Aus den ermittelten Gleichgewichtskonstanten folgt nämlich, daß es kein $p_{\mathrm{H}}$-Gebiet gibt, bei dem $\mathrm{FH}^{++}$in nennenswerter Menge hinreichend lange beständig ist: Eine Rechnung ergibt, daß beim totalen Gleichgewicht im günstigsten Falle (beim $p_{\mathrm{H}} 2,58$ ) der Anteil des $\mathrm{FH}^{++}$ nur 5\% der Fuchsin-Bruttokonzentration beträgt.

5. Abschließend kann also gesagt werden, daß die mitgeteilten Versuchsergebnisse weitere experimentelle Beweise für das Vorliegen der Mesomerie bei den Triphenylmethanfarbstoff-Kationen liefern.

Wir danken Frl. L. Gellrich und Hrn. Schuchardt für ihre gewissenhafte Mithilfe bei den Messungen. 